\title{
Fixação suspensiva percutânea de luxação crônica da articulação radioulnar distal (ARUD) usando um dispositivo de endobutton de laço ajustável: Relato de caso*
}

\section{Percutaneous Suspensory Stabilization of Chronic Distal Radioulnar Joint (DRUJ) Dislocation Using an Adjustable Loop Endobutton Construct: Case Report}

\author{
Mohammed Schezan Iqbal'10 Anil Kumar Mishra ${ }^{2}$ Rajiv Kaul ${ }^{3(1)}$ \\ ${ }^{1}$ Departamento de Ortopedia, INHS Kalyani, Visakhapatnam, Índia \\ 2 Departamento de Ortopedia, Military Hospital Kirkee, Pune, Índia \\ ${ }^{3}$ Departamento de Ortopedia, Armed Forces Medical College, Pune, Índia \\ Endereço para correspondência Mohammed Schezan Iqbal, MS \\ (Orthopaedics), Department of Orthopaedics, INHS Kalyani, \\ Visakhapatnam, Andhra Pradesh, 530005, India \\ (e-mail: dr.schezaniqbal@gmail.com).
}

Rev Bras Ortop

\section{Resumo \\ Palavras-chave \\ - lesões no punho/ diagnóstico \\ - lesões no punho/ cirurgia \\ - lesões no punho/ fisiologia \\ - ortopedia/tendências \\ - fixadores externos}

Luxação crônica da articulação radioulnar distal (ARUD) foi tratada historicamente com osteotomias complexas e procedimentos reconstrutivos, geralmente resultando em rigidez intratável e perda de função. É desejável usar uma técnica de fixação que não apenas restaure a biomecânica do punho, mas também seja esteticamente atraente para o indivíduo. Apresentamos uma nova técnica de redução e fixação de uma ARUD deslocada cronicamente em um homem de 26 anos, usando uma abordagem minimamente invasiva, com restauração bem-sucedida da função da ARUD e sem complicações pós-operatórias.

Chronic distal radioulnar joint (DRUJ) dislocation has been treated historically with complex osteotomies and reconstructive procedures, often resulting in intractable stiffness and loss of function. It is desirable to use a technique of fixation that will not only restore the wrist biomechanics but also be cosmetically appealing to the individual. We present a novel technique of reduction and fixation of a chronically dislocated DRUJ in a 26-year-old male using a minimally invasive approach, with successful restoration of DRUJ function and no postoperative complications.

\footnotetext{
Keywords

- wrist injuries/ diagnosis

- wrist injuries/surgery

- wrist injuries/ physiology

- orthopedics/trends

- external fixators
}

Trabalho desenvolvido no Departamento de Ortopedia, INHS Kalyani, Visakhapatnam, Índia. recebido

11 de Abril de 2020

aceito

01 de Junho de 2020
DOI https://doi.org/

10.1055/s-0040-1715515. ISSN 0102-3616.
Copyright $\odot$ by Sociedade Brasileira de Ortopedia e Traumatologia. Published by Thieme Revinter Publicações Ltda, Rio de Janeiro, Brazil
License terms

(c) $9 \ominus$ 


\section{Introdução}

A instabilidade da articulação radioulnar distal (ARUD) ocorre devido ao rompimento tanto dos estabilizadores de punho intrínsecos (por exemplo, o complexo de fibrocartilagem triangular [CFCT]) quanto dos extrínsecos (por exemplo, os ligamentos radioulnares volar e dorsal). Isso pode ocorrer isoladamente ou em associação com fraturas do rádio distal e suas variantes (3-37\%). ${ }^{1}$ As opções de tratamento incluem osteotomias, osteoplastias, reconstruções de tecidos moles e cirurgias de salvamento, como os procedimentos de Darrach e de Sauve-Kapandi. ${ }^{1}$

No presente relatório, descrevemos uma nova fixação percutânea e suspensa para uma luxação crônica da DRUJ secundária a uma fratura antiga do rádio distal.

\section{Relato de Caso}

O paciente era um homem de 26 anos de idade, com 9 meses de história de dor e deformidade no pulso esquerdo, após aci- dente de trânsito. Na lesão inicial, houve extensa perda de pele no punho e antebraço, que foi gerenciada com um fixador externo abrangendo o punho unilateral e fixação percutânea com fio de Kirschner por um período de 2 meses. Um enxerto de pele dividido foi feito para tratar das áreas feridas mantendo o fixador externo in situ. Após a remoção do fixador, o paciente notou uma deformidade do punho que incluía uma proeminência indevida da ulna junto com uma restrição severa da pró-supinação e um clique ocasional do pulso.

No exame, o paciente apresentou deformidade do tipo manus varus, com proeminência anormal da cabeça ulnar dorsalmente. O teste de Reagan e o teste do sinal da "tecla do piano" foram positivos. Os movimentos do punho foram restritos a $50^{\circ}$ de dorsiflexão, $30^{\circ}$ de flexão palmar, $60^{\circ}$ de pronação e sem supinação. A força de preensão foi de $50 \%$ da mão normal. Não houve déficits neurovasculares na extremidade afetada.

Raios X do punho (Posteroanterior [PA] e vistas laterais) (-Fig. 1a-b) mostraram incongruência articular grosseira da articulação radiocarpal, com evidência de artrose extensa no punho e encurtamento radial precoces. Havia luxação dorsal
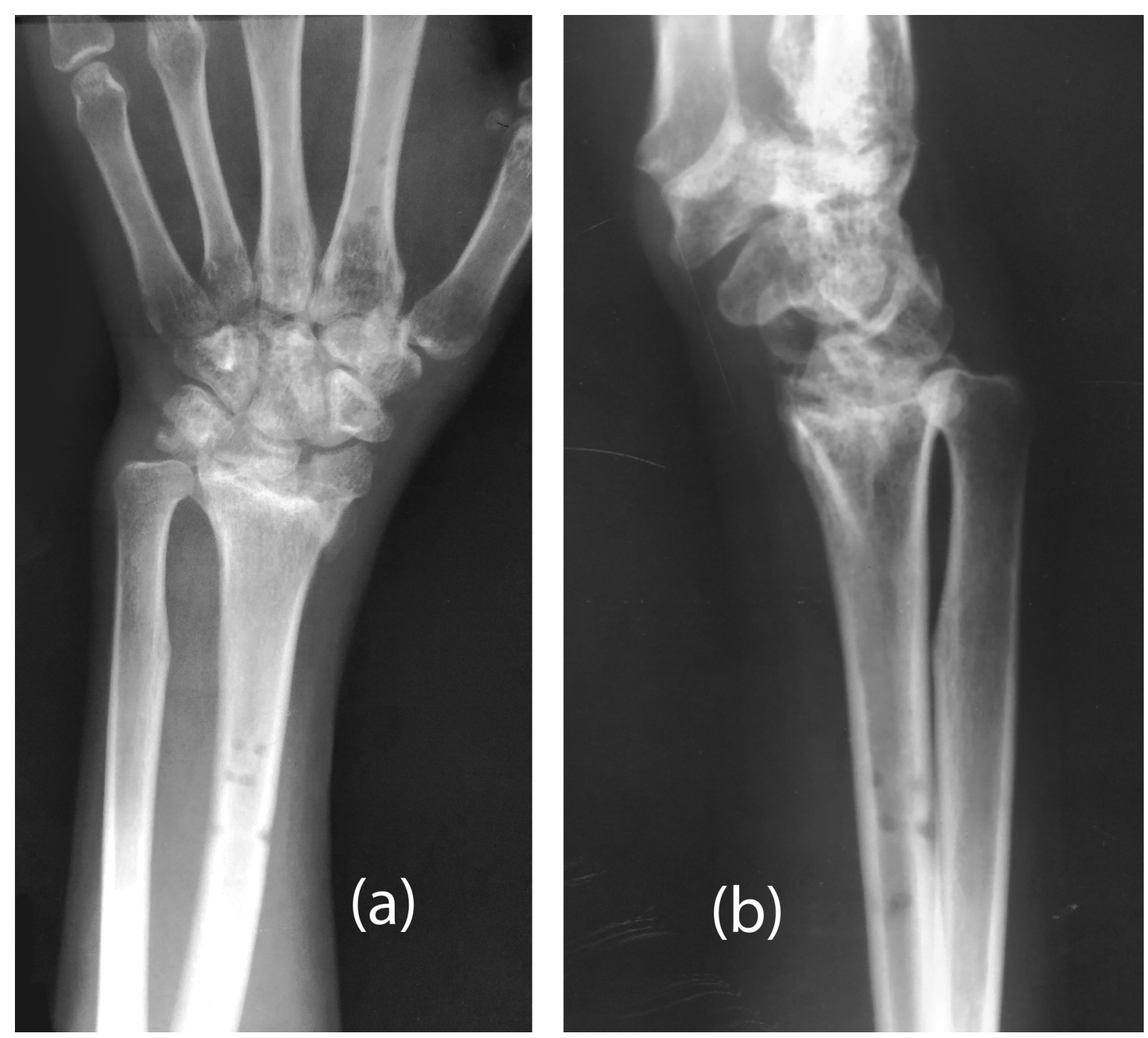

Fig. 1 Raio X pré-operatório do pulso (visualizações PA e laterais). 

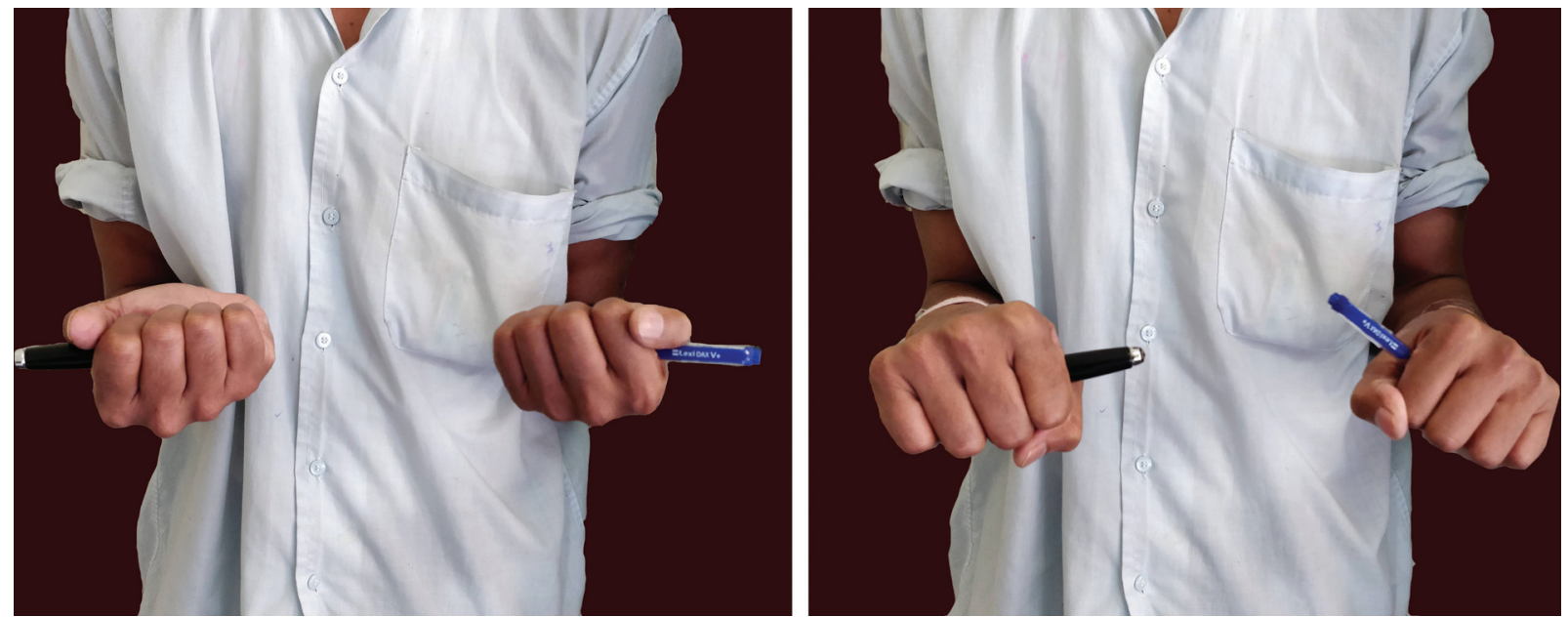

Fig. 2 Fotografia clínica pós-operatória (prono supinação).

da ulna distal na visão lateral, com sobreposição do rádio distal e da ulna na visão PA.

Tendo em vista se tratar de um paciente jovem e os requisitos funcionais, foi tomada a decisão de realizar uma intervenção cirúrgica minimamente invasiva, realizada da maneira descrita a seguir.

\section{Técnica Cirúrgica}

Sob anestesia regional, o membro superior afetado foi colocado de bruços sobre uma mesa radiolúcida. A redução fechada satisfatória da luxação DRUJ foi alcançada aplicando uma pressão direcionada volarmente na cabeça ulnar e a fixação provisória feita com um fio $\mathrm{K}$ de $1,5 \mathrm{~mm}$. Uma broca canulada de 2,7 mm foi usada sobre um fio-guia para perfurar um túnel da ulna distal ao rádio distal na direção geral do feixe oblíquo distal. Arthrex Tightrope RT (Arthrex, Inc. Naples, Florida, USA) foi passado do lado ulnar com o endobutton no lado radial e, depois de ser confirmada a redução sob fluoroscopia, o botão foi virado e apertado. A sutura de poliéster foi presa ao redor da ulna e amarrada após passar por um orifício aberto separadamente na ulna. Uma
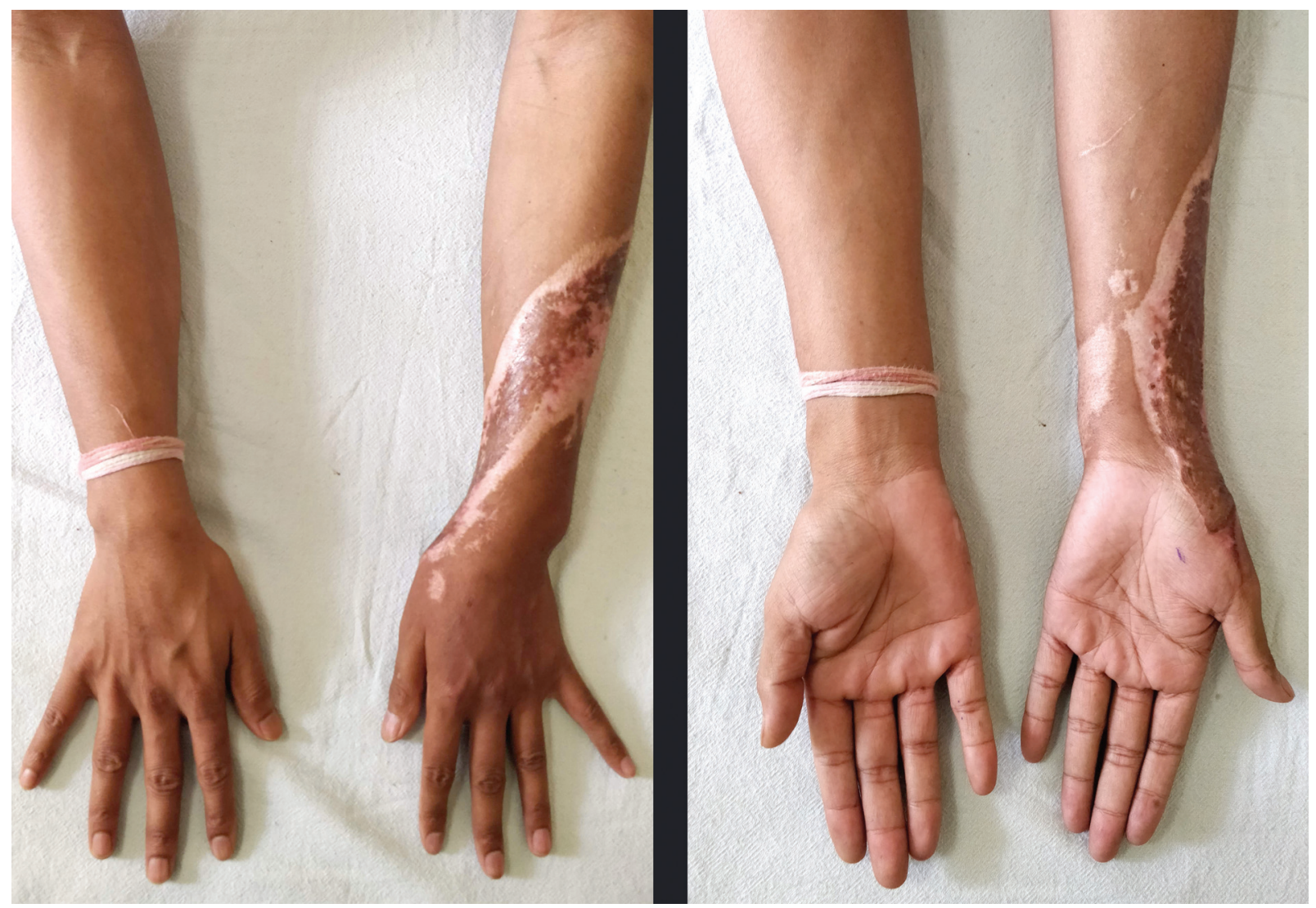

Fig. 3 Fotografia clínica pós-operatória (aspecto dorsal e volar). 
tala gessada acima do cotovelo com o punho em supinação total foi administrada por 4 semanas, após as quais foram iniciados exercícios de mobilização e fortalecimento gradual. Não tivemos complicações pós-operatórias, como parestesias, infecção, rigidez etc. A amplitude de movimento (ADM) alcançada após 3 meses de acompanhamento foi de $70^{\circ}$ de dorsiflexão, $60^{\circ}$ de flexão palmar, $80^{\circ}$ de pronação e $70^{\circ}$ de supinação. A força de preensão foi de $80 \%$ da mão normal (-Figs. 2 e 3). A radiografia pós-operatória aos 3 meses é mostrada na - Fig. $\mathbf{4 a - b}$.

\section{Discussão}

Os princípios que governam o manejo da instabilidade crônica da ARUD baseiam-se essencialmente no envolvi- mento de ossos ou tecidos moles e suas reconstruções. ${ }^{2}$ Nos casos com deformidades ósseas consideráveis, são realizadas osteotomias de realinhamento com ou sem encurtamento ulnar.

Se o CFCT for reparável, procedimentos como plicatura capsular e reinserção do CFCT usando âncoras de sutura, artroscopicamente ou abertas, geralmente são a primeira linha de tratamento. Quando o reparo direto do CFCT não é mais possível, é necessária tenodese extra-anatômica ou reconstrução ligamentar para reconstruir os ligamentos radioulnares e a inserção foveal. ${ }^{2}$ Conhecida como técnica de Adams e Berger, ${ }^{3}$ a tenodese anatômica utiliza o tendão palmar longo e necessita de túneis transósseos radiais e ulnares. Esta técnica reproduz melhor a cinemática normal da ARUD. ${ }^{4}$ Nakamura ${ }^{5}$ descreveu um procedimento extra-
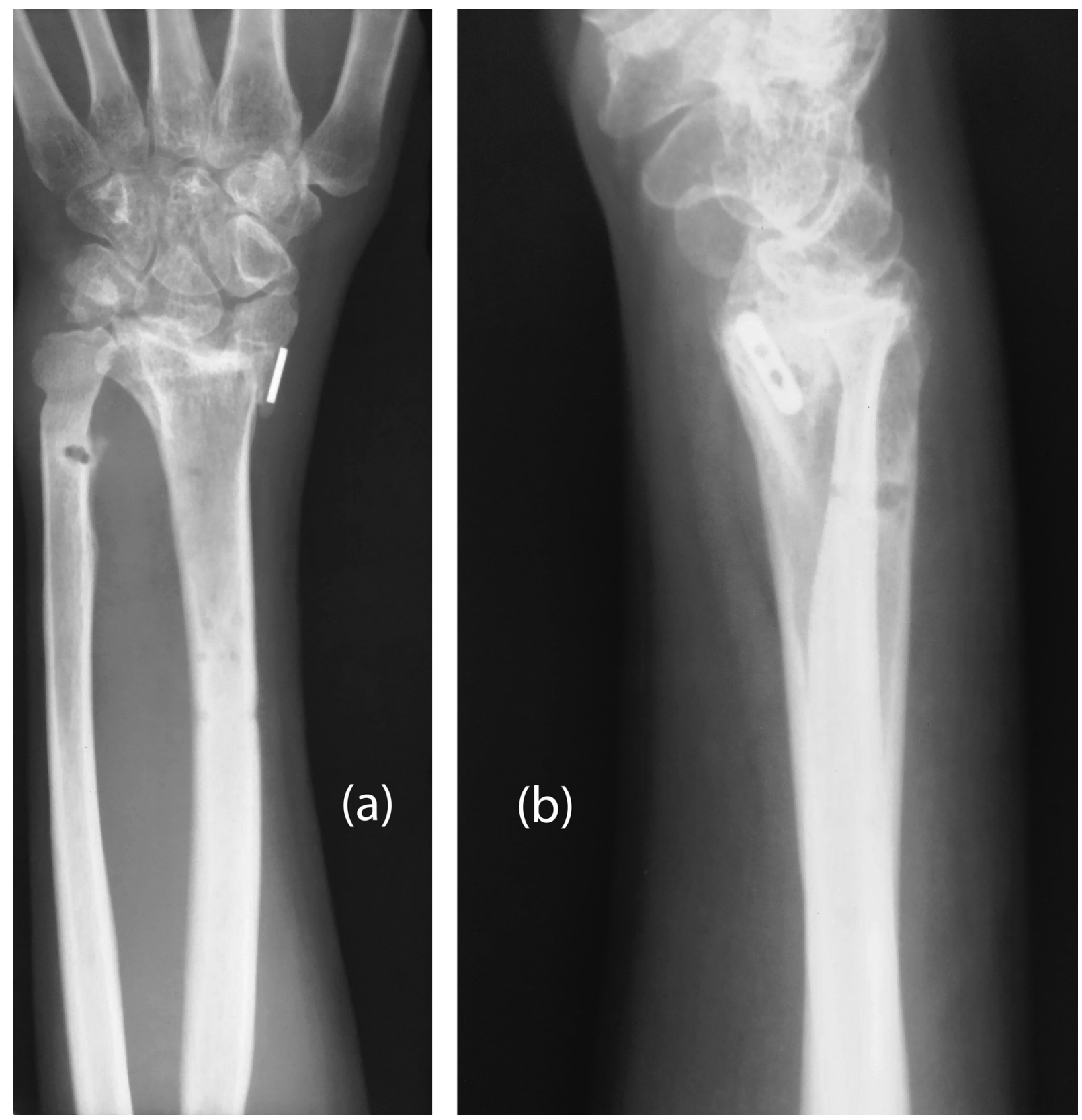

Fig. 4 Raio X pós-operatório do pulso (visualizações PA e Laterais). 
anatômico que combinou reconstrução de tenodese e ligamento usando um enxerto de hemi-extensor ulnar do carpo (EUC) de pedículo distal com um único túnel transulnar que é passado pelos remanescente do CFCT e impactado na fixação foveal. Recentemente, na reconstrução da porção distal da membrana interóssea (MIO), ${ }^{6}$ foi descrito o que configura um enxerto radioulnar isométrico que estabiliza a ARUD em toda a amplitude de movimento da pró-supinação.

Para lesões de cartilagem envolvendo a ARUD, procedimentos paliativos como Sauvé-Kapandji ${ }^{7}$ ou Darrach e Bowers apud Gaebler e McQueen ${ }^{8}$ e suas variantes podem ser executadas; no entanto, a estabilização do coto ulnar proximal continua sendo uma preocupação. Artroplastias restritas ${ }^{9}$ são limitados pelo número relativamente pequeno de estudos e pelo curto acompanhamento dessa coorte de pacientes.

Em seu trabalho, de Vries et al. ${ }^{10}$ descreveram um procedimento minimamente invasivo para estabilização da articulação radioulnar distal em espécimes cadavéricos, usando uma construção de botão de sutura colocada percutaneamente direcionada ao longo do feixe oblíquo distal da membrana interóssea. Semelhante à técnica acima, usamos uma fixação suspensiva minimamente invasiva colocada ao longo da direção da porção distal da MIO para restaurar a estabilidade da ARUD.

Nossa técnica simples é anatomicamente e biologicamente estável. A remoção pode ser feita por via percutânea, se necessário. Esta técnica é uma alternativa útil ao método tradicional de transfixação usando um fio de Kirschner. As complicações potenciais incluem diminuição da mobilidade articular e estofo excessivo da incisura sigmoide, se o dispositivo for apertado demais. Além disso, a perfuração imprecisa pode resultar em fraturas iatrogênicas da ulna ou do rádio, danos ao nervo radial superficial ou ulnar e um túnel não anatômico que impedirá os movimentos da ARUD. Com base em nossa experiência, recomendamos essa técnica no cenário de luxações agudas e crônicas da ARUD.

Conflitos de Interesse

Os autores não têm conflitos de interesse a declarar.

\section{Referências}

1 Mulford JS, Axelrod TS. Traumatic injuries of the distal radioulnar joint. Hand Clin 2010;26(01):155-163

2 Marès 0 . Distal radioulnar joint instability. Hand Surg Rehabil 2017;36(05):305-313

3 Adams BD, Berger RA. An anatomic reconstruction of the distal radioulnar ligaments for posttraumatic distal radioulnar joint instability. J Hand Surg Am 2002;27(02):243-251

4 Gofton WT, Gordon KD, Dunning CE, Johnson JA, King GJ. Comparison of distal radioulnar joint reconstructions using an active joint motion simulator. J Hand Surg Am 2005;30(04):733-742

5 Nakamura T. Anatomical Reattachment of the TFCC to the Ulnar Fovea Using an ECU Half-Slip. J Wrist Surg 2015;4(01):15-21

6 Brink PR, Hannemann PF. Distal Oblique Bundle Reinforcement for Treatment of DRUJ Instability. J Wrist Surg 2015;4(03):221-228

7 Kapandji IA. The Kapandji-Sauvé operation. Its techniques and indications in non rheumatoid diseases. Ann Chir Main 1986;5 (03):181-193

8 Gaebler C, McQueen MM. Ulnar procedures for post-traumatic disorders of the distal radioulnar joint. Injury 2003;34(01):47-59

9 Kakar S, Fox T, Wagner E, Berger R. Linked distal radioulnar joint arthroplasty: an analysis of the APTIS prosthesis. J Hand Surg Eur Vol 2014;39(07):739-744

10 de Vries EN, Walenkamp MM, Mulders MA, Dijkman CD, Strackee SD, Schep NW. Minimally invasive stabilization of the distal radioulnar joint: a cadaveric study. J Hand Surg Eur Vol 2017; 42(04):363-369 